\title{
ENSAYO DE IMPLANTACIÓN DE PASTURA EN BOSQUE DE PINO PONDEROSA
}

\author{
Buduba, $C^{1}$, Hansen, $N^{1}$, Bobadilla, $S^{1}$, Lexow, $G^{1}$ y Escalona, $M^{2}$
}

\section{RESUMEN}

Identifica al ecotono estepa bosque andino patagónico argentino un régimen hídrico de tipo mediterráneo y la presencia de vientos secos, predominantes del Oeste. La escasa oferta forrajera que actualmente brinda el ecotono está vinculada a las condiciones climáticas y a su historia de uso ganadero poco sustentable.

Las condiciones indicadas implican un bajo número de animales por hectárea, siendo intensivo el uso de los sitios que por características topográficas son más húmedos y una escasa utilización de los predominantes lugares altos y secos.

La implantación de árboles en dicha porción del paisaje podría moderar las adversas condiciones climáticas favoreciendo la presencia de forraje. El objetivo de este trabajo es presentar los resultados de una experiencia de siembra con cuatro especies forrajeras en un bosque de Pinus ponderosa implantado en el ecotono patagónico.

Fue seleccionado un rodal de 14 años ubicado en la provincia del Chubut $\left(42^{\circ}\right.$ 17' LS, $71^{\circ} 5^{\prime}$ LO), bajo condiciones de precipitación media anual de $430 \mathrm{~mm}$. Se realizó un raleo en $1.250 \mathrm{~m}^{2}$, obteniendo 667 árb ha $^{-1}, 8 \mathrm{~m}^{2}$ de área basal y $46 \%$ de apertura de canopeo. En un suelo areno franco profundo se retiraron los residuos forestales y el incipiente horizonte orgánico, pasando luego un rastrillo de siembra.

En julio de 2009 se dispuso, en parcelas de 1,44 $\mathrm{m}^{2}$, un diseño aleatorizado con 14 tratamientos $(n=9)$, conformados por cuatro especies (alfalfa, trébol rojo, agropiro, festuca) solas o combinadas. Al final del verano (período seco) se realizó un recuento de plantas en donde el agropiro se diferenció significativamente del resto (ANVA, Tukey, $\mathrm{p}<0,05)$, con un promedio de 39 plantas $\mathrm{m}^{-2}$. El agropiro es una gramínea perenne cultivada en zonas con condiciones edafoclimáticas limitantes que podría representar una importante mejora forrajera.

Esta experiencia es parte de un proyecto orientado a mejorar la oferta forrajera en el ecotono, utilizando al bosque de pino como facilitador.

Palabras clave: Siembra, estepa, bosque, pastura.

1 INTA Esquel, Chubut, Argentina. cgbuduba@correo.inta.gov.ar

2 Facultad de Ingeniería, UNPSJB, Esquel, Chubut, Argentina. 


\section{SUMMARY}

The ecotone steppe andino patagónico forest is under a mediterranean climate regime, with westerly dry winds. Climate and overgrazing history resulted in a low carrying capacity, with intensive grazing on meadows and a relative low use of higher and dryer slopes.

Tree plantation on these sites could moderate the effect of harsh climate conditions leading to improved grass offer.

This paper presents results obtained with four species (alfalfa, red clover, fescue and wheatgrass) under a 14 years old Pinus ponderosa forest in the ecotone zone.

The site is located in the Chubut province of Argentina (42ำ $17^{\prime} \mathrm{SL}, 7^{\circ} 5^{\prime} \mathrm{WL}$ ) with $430 \mathrm{~mm}$ of annual rain average. The forest was thinned to 667 trees ha ${ }^{-1}, 8 \mathrm{~m}^{2}$ of basal area and $46 \%$ of open canopy.

A place on a deep sandy loam soil was cleared and, on July 2009, 14 plots were seeded. Each plot covered $1.44 \mathrm{~m}^{2}$ and were alocated at random $(\mathrm{n}=9)$ with the four species alone or mixed. At the end of summer (dry period) seedlings were counted.

Wheatgrass counting were higher than the other species $(p<0,05)$ with an average of 39 seedlings $\mathrm{m}^{-2}$. Weathgrass is a perennial grass well adapted to dry conditions that could improve the forage offer under these conditions.

This work is part of a project leading to improve forage production on the ecotone under pine forest cover.

Key words: Sowing, steppe, forest, pasture. 


\section{INTRODUCCIÓN}

El ecotono estepa bosque andino patagónico argentino es utilizado para la producción ganadera extensiva. En un ambiente definido por un régimen hídrico de tipo mediterráneo y vientos secos predominantes del Oeste, el pastizal es el principal recurso natural que sustenta la actividad ganadera. Sin embargo, más de 100 años de uso han deteriorado el recurso, que hoy evidencia en amplias superficies serios procesos de degradación, disminuyendo la receptividad de animales por hectárea (Salomone et al., 2008).

La implantación de árboles en el mismo paisaje es alentada desde hace unos 40 años por diferentes organismos técnico - científicos como una alternativa productiva que puede generar diversos beneficios socio ambientales. Esto generó la existencia de parches boscosos en una matriz de pastizal, que en la provincia del Chubut suman unas 21.500 ha, predominando los rodales con menos de 15 años (Bava et al., 2006). La superficie de cada bosque es variable y en general tienen escaso manejo silvícola, siendo muy susceptibles a los daños por plagas y fuego.

Dadas las actuales características de numerosos rodales sin manejo, que presumiblemente mejorarían las condiciones micro ambientales para la implantación de especies herbáceas al reparo del viento, es factible realizar intervenciones que mejoren la calidad de la madera y la oferta forrajera.

En este marco se inició en el año 2009 un proyecto que estudia la factibilidad de implementar sistemas agroforestales en el ecotono de la provincia del Chubut.

El objetivo de este trabajo es mostrar los resultados de una experiencia de siembra con cuatro especies forrajeras en un bosque de Pinus ponderosa Dougl. ex Laws. implantada en el ecotono patagónico argentino.

\section{METODOLOGÍA}

Se seleccionó un rodal de pino ponderosa en la provincia del Chubut (42ำ17' lat. S, $71^{\circ} 5^{\prime}$ long. O), con una precipitación media anual de $430 \mathrm{~mm}$, una superficie total de 9 ha, 14 años de edad y 5,8 $\mathrm{m}$ de altura de los árboles dominantes.

Se realizó un raleo entre filas (sistemático) en $1.250 \mathrm{~m}^{2}$, obteniendo 667 árb ha-1, $8 \mathrm{~m}^{2}$ de área basal y $46 \%$ de abertura del dosel (originalmente 2.000 árb ha- ${ }^{1}, 17 \mathrm{~m}^{2}$ de área basal y $26 \%$ de abertura del dosel).

En las filas remanentes también se extrajeron árboles, luego se podaron todos los ejemplares hasta $2 \mathrm{~m}$ y se retiraron manualmente los residuos forestales, inclusive el incipiente horizonte orgánico. El suelo era areno franco, profundo $(+110 \mathrm{~cm})$, con pH (1:1) 7,15 y $1,8 \%$ de materia orgánica total en los $20 \mathrm{~cm}$ superficiales.

En julio de 2009 se dispusieron parcelas de 1,44 $\mathrm{m}^{2}$ en un diseño aleatorizado con 14 tratamientos $(n=9)$ : 
1 - Agropiro / alfalfa (12 y $5 \mathrm{~kg} \mathrm{ha}^{-1}$ );

2 - Festuca / trébol rojo (6 y $\left.6 \mathrm{~kg} \mathrm{ha}^{-1}\right)$;

3 - Festuca / alfalfa (6 y $\left.5 \mathrm{~kg} \mathrm{ha}^{-1}\right)$;

4 - Agropiro (20 kg ha-1);

5 - Alfalfa (12 $\left.\mathrm{kg} \mathrm{ha}^{-1}\right)$;

6 - Trébol rojo (12 kg ha-1);

7 - Festuca $\left(10,5 \mathrm{~kg} \mathrm{ha}^{-1}\right)$;

8 - Agropiro / alfalfa pelleteado (12 y $5 \mathrm{~kg} \mathrm{ha}^{-1}$ );

9 - Festuca / trébol rojo pelleteado (8 y $\left.6 \mathrm{~kg} \mathrm{ha}^{-1}\right)$;

10 - Festuca / alfalfa pelleteado (6 y $5 \mathrm{~kg} \mathrm{ha}^{-1}$ );

11 - Agropiro pelleteado (20 $\left.\mathrm{kg} \mathrm{ha}^{-1}\right)$;

12 - Alfalfa pelleteado (12 $\left.\mathrm{kg} \mathrm{ha}^{-1}\right)$;

13 - Trébol rojo pelleteado (12 $\left.\mathrm{kg} \mathrm{ha}^{-1}\right)$

14 - Festuca pelleteado $\left(10,5 \mathrm{~kg} \mathrm{ha}^{-1}\right)$.

Los tratamientos pelleteados consistieron en pequeñas esferas de arcilla mezclada con semillas, dispuestas sobre la superficie del suelo según la propuesta de Fukuoka (2009). Se utilizó un rastrillo para marcar 4 surcos de siembra, cubriéndose con tierra sólo los primeros siete tratamientos (siembra tradicional).

Al final del verano (abril 2010) se realizó un recuento de plantas en cada parcela considerando los 2 surcos centrales. Para evaluar diferencias entre tratamientos se realizó un análisis de varianza (Tukey, $p<0,05$ ).

\section{RESULTADOS Y DISCUSIÓN}

A los 9 meses de la siembra, al concluir el período seco, el agropiro sembrado en surcos, con una densidad de $20 \mathrm{~kg} \mathrm{ha}^{-1}$ (tratamiento 4), logró el mayor número de plantas establecidas (Cuadro No 1).

El siguiente tratamiento con mayor número de plantas se observó con agropiro / alfalfa, aunque la densidad para la primera especie sólo era de $12 \mathrm{~kg} \mathrm{ha}^{-1}$. Los tratamientos pelleteados (esferas de arcilla y semillas), que según Fukuoka (2009) podrían ser una alternativa para la implantación de pasturas en lugares secos y degradados, no resultaron ser significativamente adecuados. 
El agropiro es una gramínea perenne cultivada en zonas con condiciones edafoclimáticas limitantes que necesita de un manejo adecuado para evitar que se encañe (los tallos lignificados son poco palatables). A bajas densidades el agropiro forma matas (menor calidad de forraje), no obstante, lograr densidades de 39 plantas $\mathrm{m}^{-2}$ podría representar una importante mejora forrajera en el ecotono patagónico.

CUADRO N ${ }^{\circ} 1$

NÚMERO DE PLANTAS PROMEDIO POR ESPECIE Y TRATAMIENTO

\begin{tabular}{|c|c|c|c|}
\hline Tratamiento & $\begin{array}{c}\text { Número } \\
\text { de plantas } \\
\text { promedio por } \\
\text { especie }\end{array}$ & $\begin{array}{c}\text { Número } \\
\text { de plantas } \\
\text { promedio por } \\
\text { tratamiento }\end{array}$ & $\begin{array}{c}\text { Número } \\
\text { de plantas } \\
\text { promedio por } \\
\text { m2 }\end{array}$ \\
\hline 1 - Agropiro $^{3}$ / alfalfa $^{4}$ & $10 / 0$ & $10,11^{\mathrm{b}}$ & 14 \\
\hline 2 - Festuca ${ }^{5} /$ trébol rojo $^{6}$ & $4 / 0$ & $4,22^{b c}$ & 6 \\
\hline 3 - Festuca ${ }^{5} /$ alfalfa $^{4}$ & $1 / 0$ & $0,89 \mathrm{bc}$ & 1 \\
\hline 4 - Agropiro $^{3}$ & 28 & $28,22^{a}$ & 39 \\
\hline 5 - Alfalfa $^{4}$ & 1 & $1,22^{b c}$ & 2 \\
\hline 6 - Trébol rojo 6 & 0 & $0^{c}$ & 0 \\
\hline 7 - Festuca 5 & 3 & $2,67^{b c}$ & 4 \\
\hline 8 - Agropiro $^{3}$ / alfalfa ${ }^{4}$ pelleteado & $4 / 2$ & $5,56^{b c}$ & 8 \\
\hline 9 - Festuca ${ }^{5}$ / trébol rojo ${ }^{6}$ pelleteado & $2 / 1$ & $2,22^{b c}$ & 3 \\
\hline 10 - Festuca ${ }^{5} /$ alfalfa $^{4}$ pelleteado & $5 / 1$ & $6,33^{b c}$ & 9 \\
\hline 11 - Agropiro ${ }^{3}$ pelleteado & 7 & $7^{\mathrm{bc}}$ & 10 \\
\hline 12 - Alfalfa ${ }^{4}$ pelleteado & 2 & $2,22^{b c}$ & 3 \\
\hline 13 - Trébol rojo ${ }^{6}$ pelleteado & 0 & $0,22^{C}$ & 0 \\
\hline 14 - Festuca ${ }^{5}$ pelleteado & 4 & $3,56^{b c}$ & 5 \\
\hline
\end{tabular}

(Letras diferentes manifiestan diferencias significativas $p<0,05$ )

3 Thynopirum ponticum.

4 Medicago sativa.

5 Festuca arundinacea.

6 Trifolium pratense. 


\section{CONCLUSIONES}

Concluida una estación de crecimiento el agropiro mostró el mejor comportamiento al ensayar cuatro especies forrajeras para implantar una pastura en bosque de pino ponderosa.

Este resultado es parte de un proyecto orientado a mejorar la oferta forrajera en el ecotono, utilizando al bosque de pino como facilitador.

En este marco es necesario seguir investigando nuevas especies forrajeras, profundidad y época de siembra, variantes en la elaboración de esferas de arcilla y niveles de cobertura arbórea para el adecuado establecimiento.

Se prevé continuar durante los próximos años hasta lograr resultados a nivel predial y de esa manera, generar una herramienta que, al incentivar el manejo silvícola, mejore la calidad de la madera y la oferta forrajera del bosque.

\section{AGRADECIMIENTOS}

Al Señor Juan Calfupán por la confianza demostrada al permitir trabajar en su predio. A Gustavo Ocampo, Sergio Binda, Georgina Ciari y Cecilio Jones, un sincero reconocimiento por los valiosos aportes técnicos y por la ayuda en los trabajos de campo.

\section{REFERENCIAS}

Bava, J, J. D Lencinas, A. Haag, G. Aguado, 2006. Los bosques del Chubut. Distribución, estado y potencial para la producción maderera. Reporte basado en los resultados del proyecto: "Determinación de la materia prima disponible para la realización de proyectos de inversión forestales en las cuencas de Chubut", Asistencia técnica del CFI, DGB y $\mathrm{P}$ de la provincia del Chubut. 76 p. Disponible en http://www.chubut.gov.ar/srnma/ archives/030847.php?id=-1.

Fukuoka, M., 2009. La senda natural del cultivo. Regreso al cultivo natural. Teoría y práctica de una filosofía verde. Ed. Comunitaria Mallín Ahogado, El Bolsón, Río Negro, Argentina. $300 \mathrm{p}$.

Salomone, J, M. E Llanos, A. San Martín, N. O Elissalde, S. Behr, 2008. Uso del suelo y degradación de tierras en la Provincia del Chubut. Evolución en los últimos veinte años. Actas XXI Congreso Argentino de la Ciencia del Suelo. Potrero de los Funes, San Luis, Argentina. 